\title{
On the utility of the jnd in predicting motor retention: An initial consideration
}

\author{
PHILIP H. MARSHALL, VALENCIA W. MILLS, and ROBERT T. SWANTON \\ Texas Tech University, Lubbock, Texas 79409
}

\begin{abstract}
Subjects attempting to reproduce a linear displacement response are viewed as moving until they feel that their attempted response is correct. This paper presents the argument that a quantitative predictor of their reproduction can be made in terms of the psychophysical notion of the jnd. Experiment 1 obtained an empirical estimate of the jnd for a linear displacement response. Experiment 2 was a reanalysis of the motor recognition study of Marshall (1972), from which a jnd estimate was also computed. The corresponding estimates were remarkably close and were able to predict the "recall" data from that same study.
\end{abstract}

When subjects perform in a motor retention study, they are often expected to reproduce a standard or criterion movement previously experienced. Psychophysically, that task is identical to having subjects make an equality judgment, varying one stimulus so that it is equal to a standard. In the case of motor retention studies, the standard is an internal representation (perceptual trace, schema) of the movement, rather than an external standard, as is usually encountered in psychophysical studies. Also, the variables of interest in retention studies usually affect the comparison process between the attempted equality judgment and the standard (e.g., number or repetitions, retention interval, varieties of feedback, potentially interfering movements, etc.). The similarities between retention and psychophysical methodologies for motor responses are particularly evident because, unlike the situation in most verbal retention studies, the input (acquisition) and output (retention) modalities of the task are exactly the same. One should be able to observe the operation of psychophysical concepts and mechanisms, and they should have a place in our motor retention theories.

From the subject's point of view, a motor response is attempted until the subject detects or recognizes the correct movement extent. In essence, the subject moves until he realizes that a correct response has occurred, that is, correct according to degree of success of the match between the reference mechanism and incoming sensory feedback. Adams (1971, p. 15) has stated, concerning the degree of subjective and objective error, that "one moves until he 'knows' he is correct," and Schmidt (1975, p. 241) has made a similar statement: "The subject is moving to that location that he recognizes as being correct."

Psychophysics, then, gives us the concept that when a subject concludes an attempted response, the extent of

Reprint requests should be sent to Philip H. Marshall, Department of Psychology, Texas Tech University, Lubbock, Texas 79409. error falls within a just-noticeable difference, or jnd. Indeed, any motor memory reproduction task is asking the subject to make an equality judgment, and the instructions could just as easily be presented in psychophysical terms: "Move the slide until it is equal to the standard extent to which you just moved." Experiment 1 empirically and directly obtained an estimate of the jnd for a simple linear positioning task and also considered direct "recall" attempts of a criterion movement vis-à-vis the jnd estimate. In Experiment 2, the data from the motor recognition memory study of Marshall (1972) were reanalyzed as if subjects had performed a psychophysical task, and, according to psychophysical assumptions, an estimate of the jnd was computed and compared with that actually obtained in Experiment 1.

\section{EXPERIMENT 1}

\section{Method}

Subjects. The subjects were 21 right-handed males who volunteered to fulfill a course requirement.

Apparatus. The apparatus was a version of the common linear displacement apparatus. A slide with a handle mounted on top ran nearly without friction along a stainless steel track. A second slide moving from the opposite direction could be locked in place and, when met by the subject's slide, defined a specific movement extent.

Design. Three conditions of movement were required for each subject, and each condition began with a movement to the same defined target location of $25 \mathrm{~cm}$. In one condition, subjects responded as in the usual motor reproduction task, moving to the "exact" location previously experienced. The other two conditions were used to obtain direct estimates of the jnd. In one, subjects were instructed to move to a location just noticeably longer than the target, and in the second, to a location just noticeably shorter than the target.

Procedure. Subjects were seated in front of the apparatus such that they could comfortably reach the grip on the slide with their right hand. They were blindfolded throughout the experiment except during the reading of the instructions.

At the command "grip," the subject moved the slide at a moderate deliberate speed, with one continuous movement, until the stop slide was contacted. The subject then returned his hand to his lap, and the experimenter moved the stop out of the way and reset the slide to the original starting position. After a 
$10-\mathrm{sec}$ retention interval, the experimenter gave one of three commands indicating what the nature of the subject's next movement should be: "just short," "just long," or "exactly" to the target. The subject's response was recorded as algebraic error in millimeters. Thus, a trial was defined as a movement to the target and then a second movement under one of the three conditions. Each trial was separated by a 10 -sec intertrial interval. Subjects received one of five random orders of trials. Each order was organized into 10 blocks of six trials each, with each of the three conditions occurring twice randomly within a block. This procedure provided 20 observations from each subject under each of the three conditions.

\section{Results and Discussion}

Figure 1 presents all the basic data from this study. Performance under each condition is shown in this figure with reference to zero error. Movement under the "exact" condition is represented by the three inner curves. Algebraic error under this condition shows a trend toward more positive error over blocks. Absolute error is also shown for the "exact" condition on those movements that were either long or short of the criterion length $(25 \mathrm{~cm})$. It can be observed that there was essentially no change over blocks in the amount of error, given that the subjects' attempts were either long or short. Thus, the shift toward more positive algebraic error over block was due to more subjects' making this type of error ( $81 \%$ on Block 10 vs. $62 \%$ on Block 1$)$.

The data depicted for the "just long" and "just short" attempts are only for those attempts that were made in the appropriate direction. Under the "just long" condition 95\% and under the "just short" condition $83 \%$ of the attempts were in the correct direction. The most parsimonious explanation for the greater percentage of correct-direction "just long" attempts is the less restricted range of movement past the stop. This would also account for the greater mean estimate of a "just long" attempt ( $46.21 \mathrm{~mm}$ past the target) compared to that of a "just short" attempt (33.72 mm short of the target).

The means of the "just long" and "just short" attempts can be taken as direct estimates of the jnd.

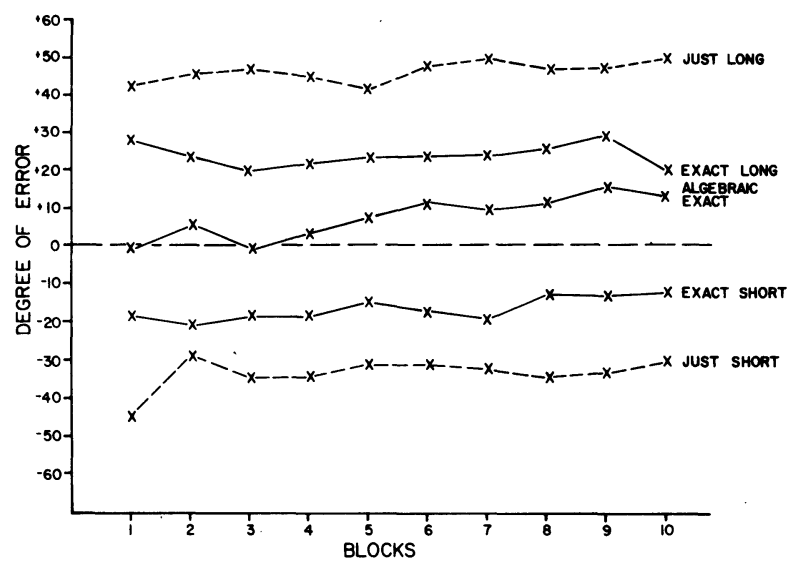

Figure 1. Mean performance over blocks of the three conditions for Experiment 1.
The average of the two, then, provides the best estimate, and that value was $39.97 \mathrm{~mm}$ under the present conditions. Noteworthy at this point is that the average long error and average short error responses under the "exact" condition fell almost exactly at the midpoints of the "just long" and "just short" jnd intervals (23.25 $\mathrm{mm}$ and $16.86 \mathrm{~mm}$, respectively). On average, then, the amount of error on these long and short attempts under the "exact" condition can be said to have fallen within the respective jnd estimates.

\section{EXPERIMENT 2}

The purpose of this experiment was to compare the obtained estimates of jnd from the first study with post hoc derived estimates from earlier reported data (Marshall, 1972). Admittedly, this is not a new study, but we will treat it as such for purposes of exposition and clarity. The reader is referred to the original version for full details. It should be made clear that the study was a motor recognition study and was not concerned at the time with psychophysical considerations; nor were subjects required to perform the task as it is reconceptualized below.

\section{Method}

Subjects. Participating were 240 right-handed undergraduate males fulfilling a course requirement.

Apparatus. The apparatus was similar to that used in Experiment 1.

Design. A two-alternative forced-choice recognition task was used, with the independent variables being (1) the number of repetitions before the retention interval (one or six), (2) the duration of the retention interval $(5,60$, or $90 \mathrm{sec})$, and (3) four levels of the discriminability of alternatives $( \pm 5 \%, \pm 10 \%, \pm 15 \%$, $\pm 20 \%$ ) in a completely between-subjects design. While the results of the Marshall (1972) study found significant influence on recognition performance for all of these variables, the present reconceptualization of that study will be concerned only with performance under the one-repetition condition, at the $5-\mathrm{sec}$ retention interval. Thus the data to be reconsidered are based on the performance of 40 subjects.

Procedure. Subjects moved the slide to a predefined stop, waited $5 \mathrm{sec}$, and then responded to the recognition test. On each of two test trials, either the criterion movement or one longer or shorter movement was given, and the subject's actual task was to tell which of the two test movements was the same as the initially presented criterion. The proportion of correct responses under each alternative condition was obtained, with the score being used based on 10 trials/subject (for any alternative condition, subjects had 10 trials with a long and 10 trials with a short alternative movement paired with the criterion).

\section{Results and Discussion}

The reevaluation mentioned above concerns reconceptualizing the study as a modified method-of-constantstimuli psychophysical procedure. To begin, and remember that this was not actually the case, consider that each pair of test trials had required the subject to make a judgment as to which movement was longer. On a test trial sequence with a longer alternative length, the subject, if giving a correct response, judged the longer alternative as being longer. On a test sequence with a shorter 
alternative, a correct response indicated the criterion was judged longer. For the purposes of a psychophysical comparison, however, we would be interested in obtaining data on estimates of "longer than" judgments under each alternative condition. The "longer than" judgments may be obtained directly for the long-alternative trials as the proportion of correct responses. To obtain a comparable measure from the short-alternative trials (i.e., the proportion of times the shorter length was judged to be longer), we must subtract the proportion of correct responses from 1.00 .

Based on the above method, Figure 2 presents the derived psychophysical function showing the percentage of times alternatives of $\pm 5 \%, \pm 10 \%, \pm 15 \%$, and $\pm 20 \%$ were judged longer than the criterion (the data are reported as the average of the criterion lengths used, $220 \mathrm{~mm}$ ). The criterion was never paired with itself, so there is a break at the " $0 \%$ difference" point.

To obtain an estimate of the jnd from these data, the following method was used (Kurtz, 1965). Points on the abscissa corresponding to ordinate values of $0 \%$ and $100 \%$ represent values of the comparison stimuli that would have yielded perfect discriminability from the criterion (standard). The abscissa value corresponding to an ordinate value of $50 \%$ would have indicated the value of the comparison stimulus that would have yielded maximum confusability with the standard. ${ }^{1}$ Halfway between the intervals $0 \%-50 \%$ and $50 \%-100 \%$ would represent uncertainty half of the time, and the stimulus values associated with those midpoints would each provide an estimate of the jnd. It is necessary to know, then, those stimulus values corresponding to $25 \%$ and $75 \%$ accuracy. To obtain those values, simple arithmetic interpolation was used between the known values on Figure 2. The average of those two values was $15.84 \%$, or $34.85 \mathrm{~mm}(.1584 \times 220 \mathrm{~mm})$, as the derived estimate of the jnd.

Referring to the obtained estimate of the jnd from Experiment $1(39.97 \mathrm{~mm})$, the present derived estimate of $15.84 \%$ would have predicted a jnd of $39.60 \mathrm{~mm}$ $(.1584 \times 250 \mathrm{~mm})$ for Experiment 1, a difference of only $.37 \mathrm{~mm}$. This correspondence is remarkably close, given that in at least one respect, the conditions of the two studies were not identical: a 10 -sec retention interval in Experiment 1 and a 5-sec interval in Experiment 2. The reliability of the estimate obtained in Experiment 1 is confirmed by the reconceptualization and analysis of the older data.

The original study from which the derived jnd was calculated (Marshall, 1972) also provided data on a standard "recall" task. The average absolute error under a comparable one-repetition, 5-sec retention interval condition was $18.21 \mathrm{~mm}$. Using the observation from Experiment 1 that the subjects responded under the "exact" condition on long and short attempts at the midpoint of the jnd interval, on the average, the predicted amount of average absolute error for the Marshall (1972) recall data is $17.43 \mathrm{~mm}(34.85 \mathrm{~mm} \div 2)$, a difference of only $.78 \mathrm{~mm}(18.21 \mathrm{~mm}-17.43 \mathrm{~mm})$.

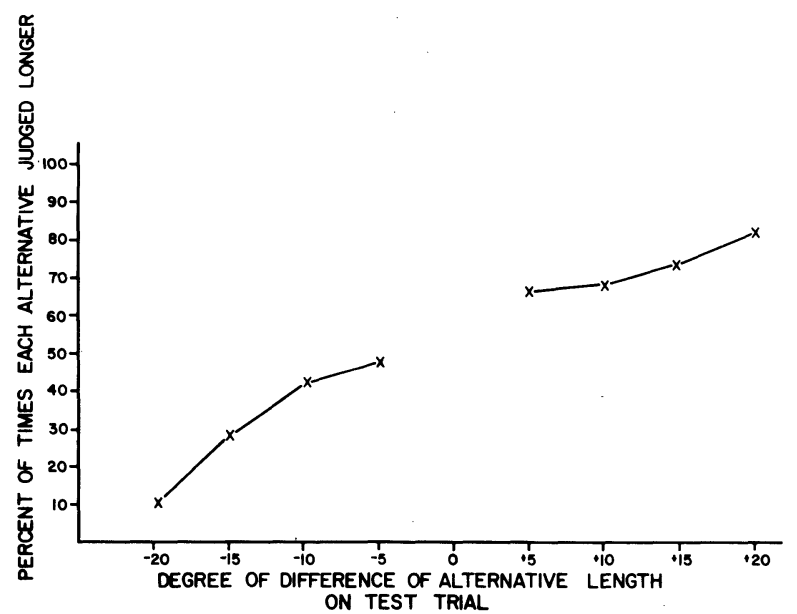

Figure 2. The psychophysical reanalysis of the Marshall (1972) recognition data: percent of "longer than" judgments given to each type of alternative.

\section{GENERAL DISCUSSION}

A conclusion from the exercise just completed is that the concept of the jnd can provide a useful quantitative predictive component absent in theories of retention of displacement responses. Such a conclusion does not address the conceptual validity of the mechanisms maintained by the theories, but it may prove to be a quantitative mechanism for them. It may be possible to criticize the use of a concept (jnd) that has been virtually replaced by signal detection theory considerations in contemporary psychological research. However, the intention of this study was to demonstrate that an experimentally derived measure that has a certain amount of face validity for what subjects are assumed to be doing on simple displacement tasks may have some usefulness in predicting their retention scores. The actual prediction of retention accuracy has not been a concern for motor retention research, and the present data may be the impetus for research of that kind.

\section{RETERENCES}

Adays, J. A. A closed-loop theory of motor learning. Journal of Motor Behavior, 1971, 3, 111-150.

KUntz, K. H. Foundations of psychological research. Boston: Allyn a Bacon, 1965.

Marahall, P. H. Recognition and recall in short-term motor memory. Journal of Experimental Psycholozy, 1972, 95, 147. 153.

Schmidr, R. A. A schema theory of discrete motor skill learning. Psycholosical Review, 1975, 82, 225-260.

\section{NOTE}

1. The method of constant stimuli, upon which this reconceptualization of the data rests, requires that on some trials the standard be paired with itself. That would have meant that in the Marshall (1972) recognition study, on some test trials the criterion length should have been presented twice. That, however, was never done. To perform the post hoc derivation of the jnd from those Marshall data, the assumption is being made that the $50 \%$ point would have been at a " $0 \%$ difference" value. 